\title{
Correlation- and singular-optical approaches in diagnostics of polarization inhomogeneity of coherent optical fields from biological tissues
}

\author{
Angelsky O.V., Ushenko A.G., Angelska A.O. and Ushenko Yu.A. \\ Chernivtsi National University, 2 Kotsyubinsky St., 58012 Chernivtsi, Ukraine, \\ e-mail: yuriyu@gmail.com
}

Received: 09.04.2007

\begin{abstract}
The paper presents results related to the correlation structure of polarization images of biological tissues. The technique for measuring coordinate distribution of the degree of mutual polarization has been proposed. The topological (singular) description of polarization-inhomogeneous images of biological tissues has been analyzed. It has been shown that the average statistical size of the S-contour correlates with the half-width of autocorrelation function for the coordinate distribution of the mutual polarization degree.
\end{abstract}

Keywords: polarization singularity, scattering, biological tissue, polarization, birefringence

PACS: 42.25.Ja, 42.25.Lc, 47.63.Jd, 42.25.Fx

\section{Introduction}

As a result of interaction of coherent laser radiation with an object, the output optical field carries information on the object structure. As a rule, this information is contained in coordinate or space-frequency distributions of photometric (intensity), amplitude-phase and polarization parameters of the field. Evidently, the mechanisms of coding of this information in the output field differ substantially, depending on the nature of objects. Nevertheless, this field - let us call it as an "object field" - is an imprint of the object under test, which is reflected in the coordinate or angular distribution of one of the field parameters mentioned above.

Historically, spatial (coordinate) structure of statistical fields has been described in terms of their coherent properties, which are determined by correlations between the parallel components of the electric fields at two different positions [1]. A different type of correlation description of the object fields is based on the analysis of polarization states. It is defined by a value of maximal correlation between the orthogonal components of complex field amplitude at a given point [2]. Real optical fields are characterized by simultaneous spatial changes in both the coherence and polarization [3-5]. 
It is known that the objects (or structures) existing in nature and forming object fields can be intelligibly divided into deterministic (regular), random (statistic) and fractal (self-similar) ones. As a consequence, the set of parameters used to characterize such the structures, would differ considerably. However, a general principle formulated by Mandelbrot [6] holds true, according to which fractality of a given object should manifest itself in the fractality of parameters characterizing this object. One can suppose that the approach should be valid for all the objects and structures, including both deterministic and statistic ones, without any particular limits.

This technique will be used in the present study for the analysis of interrelation between the topological (determined in a certain sense) and statistic approaches to description of polarization structure of the object fields. As the objects of investigation, we use histological sections of birefringent human tissues (skin derma, kidney and cervix of the uterus), which are effective transformers of polarization state of optical beam [7].

\section{Polarization inhomogeneity and singularities in images of biological tissue}

It is known that, within the topological approach to the analysis of polarization structure of the object fields, it is assumed that the areas with a right circular and elliptical polarization are separated from the areas with left circular and elliptical polarizations by contours lying in the plane where polarization remains linear (S-contours) [8]. The model presentations mentioned above enable us to get an idea about the principles, algorithms and scenarios of forming polarization-inhomogeneous field structure and, moreover, to illustrate unquestionably possible mechanisms for forming this structure on a local level. The approach is based on the formalism of singly (linear) and doubly (circular) degenerated polarization singularities in electromagnetic fields [9-16]. Depending on the scale, form, distribution and repeatability frequency of such the zones, i.e. the S-contours, we obtain uniquely determined, statistical or fractal distributions of polarization states in the object field with different scales.

In this work, the model approach [3] forms a basis for analysis of polarization structure of the field scattered from biological tissues (BT). According to the model, those objects may be represented by a two-component amorphous-crystalline matrix. Optically uniaxial, birefringent BT fibrils represent polarization-transforming structures, whose properties at any point with the coordinate $r$ are described by the Jones operator

$$
\{C\}=\left\|\begin{array}{lc}
\cos ^{2} \rho+\sin ^{2} \rho \exp (-i \delta) ; & \cos \rho \sin \rho[1-\exp (-i \delta)] ; \\
\cos \rho \sin \rho[1-\exp (-i \delta)] ; & \sin ^{2} \rho+\cos ^{2} \rho \exp (-i \delta) ;
\end{array}\right\|,
$$

where $\rho$ is the angle defining direction of packing of the birefringent fibrils into the planes of BT sample. The substance of the latter adds the phase shift $\delta$ between the orthogonal components $E_{0 x}$ and $E_{0 y}$ of the laser field. According to Eq. (1), the amplitude-phase structure of BT image may be determined by the following matrix equation: 


$$
\left(\begin{array}{c}
E_{x}(r) \\
E_{y}(r)
\end{array}\right)=\{C\}\left(\begin{array}{c}
E_{0 x} \\
E_{0 y}
\end{array}\right),
$$

where $\left[E_{x}(r), E_{y}(r)\right]$ are the complex amplitudes of electric field vector of the orthogonally polarized laser oscillations at the position $(r)$ of the BT image.

Simplifying the calculations but not causing any incompleteness of the analysis, let us consider an image formed if the BT is probed with a linearly polarized laser beam, with the azimuth $\alpha=0^{0}$ relative to the plane of incidence:

$$
\left(\begin{array}{c}
E_{0 x} \\
E_{0 y}
\end{array}\right) \Rightarrow\left(\begin{array}{c}
E_{0 x} \\
0
\end{array}\right)
$$

Taking into account Eq. (3), one can rewrite Eq. (2) as follows:

$$
\begin{aligned}
& E_{x}(r)=E_{0 x}\left[\cos ^{2} \rho(r)+\sin ^{2} \rho(r) \exp (-i \delta(r))\right] \\
& E_{y}(r)=E_{0 x}[\cos \rho(r) \sin \rho(r)(1-\exp (-i \delta(r)))]
\end{aligned}
$$

In order to determine local polarization states at different points of BT image, we should make use of the corresponding coherence matrices:

$$
\{\mathrm{K}(r)\}=\left\|\begin{array}{ll}
E_{x}(r) E_{x}^{*}(r) ; & E_{x}(r) E_{y}^{*}(r) ; \| . \\
E_{x}^{*}(r) E_{x}(r) ; & E_{y}(r) E_{y}^{*}(r) . \|
\end{array}\right\|
$$

In case of elliptically polarized beam $\left(E_{0 x}+E_{o y} \exp \left(-i \delta_{0}\right)\right)$, the expressions for the amplitudes of orthogonal oscillations for optically anisotropic areas in the BT images may be presented as

$$
\begin{aligned}
E_{x}= & \cos \rho\left(E_{0 x} \cos \rho+E_{0 y} \sin \rho \exp \left(-i \delta_{0}\right)\right)+ \\
& +\sin \rho\left(E_{0 x} \sin \rho-E_{0 y} \cos \rho \exp \left(-i \delta_{0}\right)\right) \exp (-i \delta) \\
E_{y}= & \sin \rho\left(E_{0 x} \sin \rho+E_{0 y} \cos \rho \exp \left(-i \delta_{0}\right)\right)+ \\
& +\cos \rho\left(E_{0 x} \cos \rho-E_{0 y} \sin \rho \exp \left(-i \delta_{0}\right)\right) \exp (-i \delta)
\end{aligned}
$$

According to Eq. (5), coordinate distribution of the polarization azimuths $\alpha\left(r_{i}\right)$ and the ellipticities $\beta\left(r_{i}\right)$ of the $\mathrm{BT}$ image represents polarization-inhomogeneous structure:

$$
\left\{\begin{array}{l}
\alpha\left(r_{i}\right)=0.5 \arctan \left[\tan \left(2 \frac{\operatorname{Re}\left[E_{y}\left(r_{i}\right)\right]}{\operatorname{Re}\left[E_{x}\left(r_{i}\right)\right]}\right) \cos \delta\left(r_{i}\right)\right] \\
\beta\left(r_{i}\right)=0.5 \arcsin \left[\sin \left(2 \frac{\operatorname{Re}\left[E_{x}\left(r_{i}\right)\right]}{\operatorname{Re}\left[E_{y}\left(r_{i}\right)\right]}\right) \sin \delta\left(r_{i}\right)\right]
\end{array} .\right.
$$

Here $r_{i} \equiv r_{m, n}$ is the ensemble of coordinates determined by the number of pixels in 
the $\mathrm{CCD}$ camera recording the $\mathrm{BT}$ image, $\alpha$ the azimuthal angle and $\beta$ the ellipticity of light polarization at the point $(r)$.

It follows from Eq. (6) that the most probable state of polarization for the BT image is elliptical, with the ellipticity parameters given by Eq. (7). Taking Eqs. (6) and (7) into account, one can show that the conditions for singly-degenerated (linear) polarization singularities are given by the relations

$$
\begin{gathered}
\sin ^{2} \rho \cos ^{2} \rho\left(E_{0 x}^{2} \cos ^{2} \rho-E_{0 y}^{2} \sin ^{2} \rho \exp \left(-2 i \delta_{0}\right)\right) \sin ^{2} \delta=0, \\
\rho=\arctan \left[\cos \left(2 \delta_{0}\right) \frac{E_{0 y}}{E_{0 x}}\right] \\
\delta=2 q \pi(\beta=0.5 q \pi), q= \pm 0,1,2 \ldots
\end{gathered}
$$

Doubly-degenerated (circular) polarization singularities are achieved if the relation

$$
\sin ^{2} \rho \cos ^{2} \rho\left(E_{0 x}^{2} \cos ^{2} \rho-E_{0 y}^{2} \sin ^{2} \rho \exp \left(-2 i \delta_{0}\right)\right) \sin ^{2} \delta=1
$$

holds true, from which the conditions for circularly polarized points in the BT image are easily determined:

$$
\begin{aligned}
& \rho=0.25 \pi+\arctan \left[\cos \left(2 \delta_{0}\right) \frac{E_{0 y}}{E_{0 x}}\right] \\
& \delta= \pm 0.5 \pi+2 q \pi(\beta= \pm 0.25 \pi+2 q \pi), q= \pm 0,1,2,3 \ldots
\end{aligned} .
$$

Thus, the performed analysis illustrates a possibility for generating polarizationsingular points in the BT image and describes the main mechanisms for their appearance.

\section{Computer simulation of polarization structure of the object field for curvilinear birefringent fibrils}

In order to test the concepts of the above model regarding the mechanisms for forming polarization-inhomogeneous structure of the BT object field, we have performed the following simulations. Assume that the arrangement of the BT fibrils is described by a second-order curve $A_{1} x^{2}+C_{1} y^{2}+2 D_{1} x+2 E_{1} y+F_{1}=0$. Then the polarization states of the object field would undergo a continuous change at each position $r_{i}(x, y)$.

The ensemble $(\mathrm{N}=55)$ of circle-like fibrils (see Fig. 1a) has been used as virtual objects, the radii of which change with the step $\Delta R=R+0.1 R$. We have also assumed that the coordinate distribution of the phase shifts has a circle-like character (Fig. 1b). The specific values $\alpha(r), \beta(r)$ are determined by Eq. (7), where the angle $\rho$ has the meaning of angle ( $\omega=f(r)$ - see Fig. 1a) between the tangent to the curve at the point $r_{i}(x, y)$ and the direction in the sample plane.

Fig. 2 presents coordinate distributions of the ellipticity $\beta(r)$ for these model BT images for the polarization states $\left\{\alpha_{0}=45^{0} ; \beta_{0}=0^{0}\right\}$ (Fig. 2a) and $\left\{\alpha_{0}=0^{0} ; \beta_{0}=0^{0}\right\}$ 


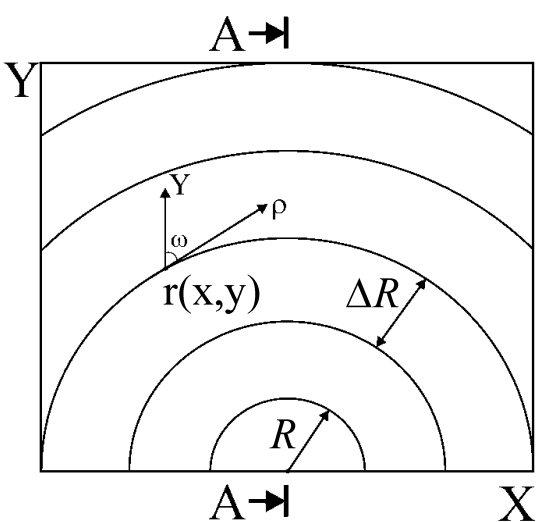

a

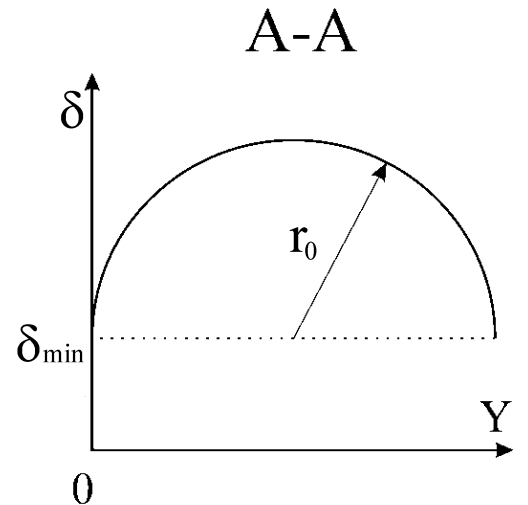

b

Fig. 1. Orientation structure of birefringent fibrils of virtual biological tissue (a) and the dependencies of spatial changing of the phase shift $\delta$ (b).

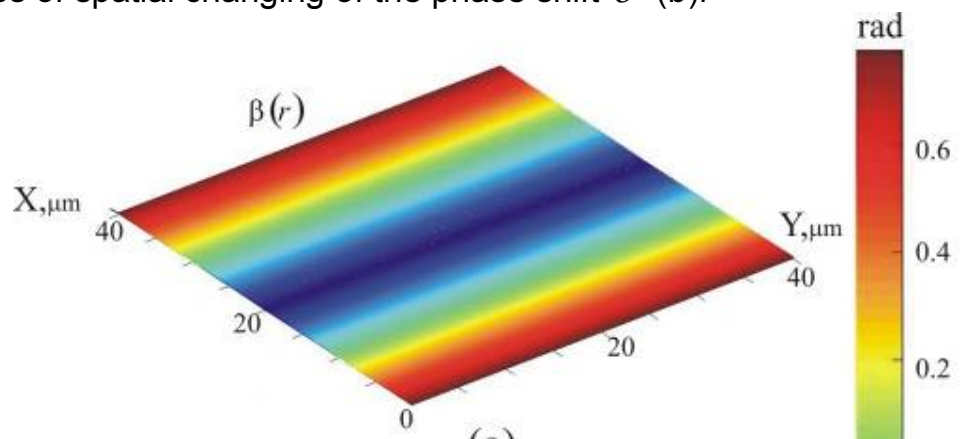

(a)

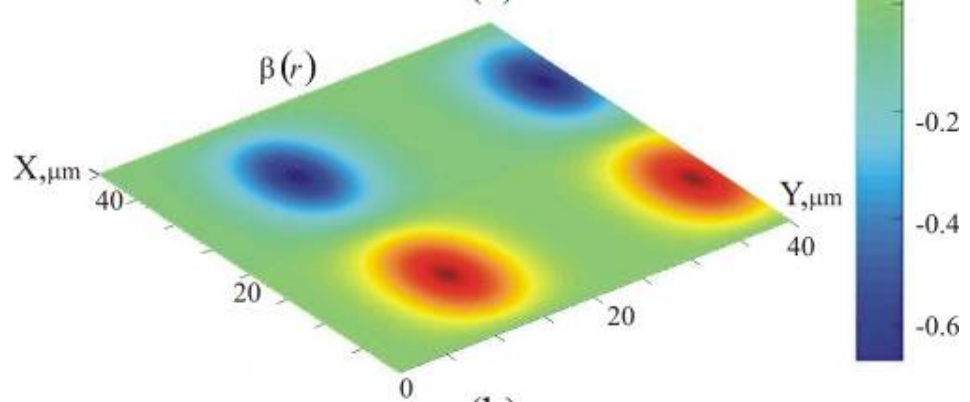

(b)

Fig. 2. Coordinate distribution of polarization ellipticities $\beta(r)$ of light oscillations for virtual biological tissue images.

(Fig. 2b). The ellipticity values are given in the RGB colour scale. It is seen that all the BT images are polarization-inhomogeneous. The polarization structure of the images presents a continuous change of elliptical polarization states determined by the coordinate distribution of singly- (S-contours, the lines $\beta(r)=0$ ) and doubly-degenerated polarization singularities (C-points, the lines $\beta(r)= \pm \pi / 4$ ). Depending on the polarization state of laser beam illuminating the ensemble of birefringent fibrils, the structure of the S-contours undergoes different changes:

- formation of closed S-contours $\left(\left\{\alpha_{0}=0^{0} ; \beta_{0}=0^{0}\right\}\right.$, Fig $\left.2 b\right)$; 
- formation of unclosed S-lines and C-lines ( $\left\{\alpha_{0}=45^{0} ; \beta_{0}=45^{0}\right\}$, Fig. 2a).

Comparative analysis of the structure of all S-contours in the images of our virtual birefringent BT reveals one common topological peculiarity: the areas with the points or lines of right circular and elliptical polarizations are separated from the areas with the left ones by the lines lying in the plane along which the polarization remains linear.

Thus, we could state that the topological singular approach proves to be adequate while analyzing mechanisms and scenarios of forming polarization structure of the object fields related to the BT with fibrillar birefringent architectonics. On the other hand, the problem of correlation interconnections between differently polarized points of the object field still remains unsolved. That is why the following section will be devoted just to this problem, for further merging the topological (singular) and the correlation approaches in the description of polarization-inhomogeneous object fields.

\section{Degree of mutual polarization of biological tissues boundary field}

A new approach has recently been suggested for the analysis of object fields mentioned above, which is based on generalization of a single-point coherence matrix $\{\mathrm{K}(r, \tau)\}$ by a two-point polarization coherency matrix $\left\{\Phi\left(r_{1}, r_{2}, \tau\right)\right\}[4,5]$ :

$$
\begin{gathered}
\{\mathrm{K}(r, \tau)\} \Rightarrow\left\{\Phi\left(r_{1}, r_{2}, \tau\right)\right\} ; \\
\|\left\langle\begin{array}{ll}
\left\langle E_{x}(r, \tau) E_{x}^{*}(r, \tau)\right\rangle & \left\langle E_{x}(r, \tau) E_{y}^{*}(r, \tau)\right\rangle \\
\left\langle E_{x}^{*}(r, \tau) E_{y}(r, \tau)\right\rangle & \left\langle E_{y}(r, \tau) E_{y}^{*}(r, \tau)\right\rangle
\end{array} \| \Rightarrow\right. \\
\Rightarrow\left\|\begin{array}{ll}
\left\langle E_{x}\left(r_{1}, \tau\right) E_{x}^{*}\left(r_{2}, \tau\right)\right\rangle & \left\langle E_{x}\left(r_{1}, \tau\right) E_{y}^{*}\left(r_{2}, \tau\right)\right\rangle \\
\left\langle E_{x}^{*}\left(r_{1}, \tau\right) E_{y}\left(r_{2}, \tau\right)\right\rangle & \left\langle E_{y}\left(r_{1}, \tau\right) E_{y}^{*}\left(r_{2}, \tau\right)\right\rangle
\end{array}\right\| .
\end{gathered}
$$

Here the symbol \langle\rangle indicates time averaging and the suffixes $x$ and $y$ depict the polarization.

According to Eqs. (12) and (13), the degree of mutual polarization (DMP) $V^{2}\left(r_{1}, r_{2}, \tau\right)$ is a main parameter determining the correlation interconnection between differently polarized points $\left(r_{1}, r_{2}\right)$ of the object field with the intensities $I\left(r_{1}, \tau\right), I\left(r_{2}, \tau\right)$ [3]. It may be written in the form [5]

$$
V^{2}\left(r_{1}, r_{2}, \tau\right)=4 \frac{v_{1}^{2}+v_{2}^{2}+v_{3}^{2}}{I\left(r_{1}, \tau\right) I\left(r_{2}, \tau\right)},
$$

where $v_{i}$ are defined by the relations

$$
\begin{gathered}
v_{1} \equiv \frac{\left\langle E_{x}\left(r_{1}, \tau\right) E_{x}^{*}\left(r_{2}, \tau\right)\right\rangle-\left\langle E_{y}\left(r_{1}, \tau\right) E_{y}^{*}\left(r_{2}, \tau\right)\right\rangle}{2} \\
v_{2} \equiv \frac{\left\langle E_{x}\left(r_{1}, \tau\right) E_{y}^{*}\left(r_{2}, \tau\right)\right\rangle+\left\langle E_{y}\left(r_{1}, \tau\right) E_{x}^{*}\left(r_{2}, \tau\right)\right\rangle}{2} \\
v_{3} \equiv i \frac{\left\langle E_{x}\left(r_{1}, \tau\right) E_{y}^{*}\left(r_{2}, \tau\right)\right\rangle-\left\langle E_{y}\left(r_{1}, \tau\right) E_{x}^{*}\left(r_{2}, \tau\right)\right\rangle}{2}
\end{gathered}
$$


These parameters are determined by the difference in contrasts of interference patterns resulting from interaction of the waves passing from the points $r_{1}, r_{2}$. They are measured for different orientations of polarization analyzer. Here $v_{1}$ corresponds to the angles $0^{\circ}$ and $90^{\circ}$ of the analyzer axes, $v_{2}$ to $45^{\circ}$ and $135^{\circ}$. The parameter $v_{3}$ should be measured by means of polarization filter, a so-called "quarter-wave plate-polarizer". Namely, the first interference pattern contrast is measured for the polarization $0^{\circ}$ and the orientation $45^{\circ}$ of fast axis of the quarter-wave plate (right circulation). The second contrast measurement corresponds to rotation of the fast axis to $135^{\circ}$, while the polarizer orientation remains unchanged. Thus, one can see that the DMP, $V^{2}\left(r_{1}, r_{2}\right)$, shows the correlation interconnection between the polarization characteristics of the field at two different positions.

Table 1 presents the DMP values for different polarization states $\vec{E}\left(r_{1}\right)$ and $\vec{E}\left(r_{2}\right)$ corresponding to two points of the object field [5]. The first row describes the case when the polarization states at the points $r_{1}$ and $r_{2}$ of the object field are linear (coplanar). The second row illustrates the case of different azimuths of linear polarizations at those points and characterizes the range of similarity of the second linear state to the first one. The third row shows interconnection between the linear and circular polarizations. Finally, the fourth row gives the DMP value for orthogonal polarization states. It is seen that $\left|V^{2}\left(r_{1}, r_{2}\right)\right|$ is equal to 1 whenever the two polarization states are identical, 0.5 for linear and circular polarizations and 0 for orthogonal polarizations.

Table 1. Examples of DMP for fully correlated fields.

\begin{tabular}{|c|c|c|}
\hline$E\left(r_{1}\right)$ & $E\left(r_{2}\right)$ & $\left|V^{2}\left(r_{1}, r_{2}\right)\right|$ \\
\hline$E_{x 1} \hat{x}$. & $E_{x 2} \hat{x}$ & 1 \\
\hline$E_{x 1} \hat{x}$. & $E_{x 2} \hat{x}+E_{y 2} \hat{y}$ & $E_{x 2}^{2} / E_{x 2}^{2}+E_{y 2}^{2}$ \\
\hline$E_{x 1} \hat{x}$. & $E_{x 2} \hat{x}+i E_{x 2} \hat{y}$ & 0.5 \\
\hline$E_{x 1} \hat{x}+E_{y 1} \exp (i \alpha) \hat{y}$ & $E_{y 1} \hat{x}-E_{x 1} \exp (i \alpha) \hat{y}$ & 0 \\
\hline
\end{tabular}

When homogeneously polarized radiation passes through a layer of birefringent BT, its characteristics are transformed, depending upon spatial distribution of optically anisotropic fibrils. In this section, we consider the possibility of direct polarization measurements for the BT image (unlike those measured using interference effects) [5], by means of determining polarization states and intensities related to different points of the boundary field.

Let us consider two differently polarized laser oscillations:

$$
\left\{\begin{array}{l}
E_{x}\left(r_{1}\right)+E_{y}\left(r_{1}\right) \exp \left(-i \delta_{1}\left(r_{1}\right)\right) \\
E_{x}\left(r_{2}\right)+E_{y}\left(r_{2}\right) \exp \left(-i \delta_{2}\left(r_{2}\right)\right)
\end{array} .\right.
$$

Here $\delta_{1}\left(r_{1}\right), \delta_{2}\left(r_{2}\right)$ are the phase shifts between the orthogonal components of the 
complex amplitudes $E_{x}, E_{y}$ at the points $r_{1}, r_{2}$. For the case of completely correlated laser image of the BT, the generalized coherence matrix given by Eq. (2) attains the form

$$
\left\{\Phi\left(r_{1}, r_{2}\right)\right\}=\left\|\begin{array}{ll}
E_{x}\left(r_{1}\right) E_{x}^{*}\left(r_{2}\right) ; & E_{x}\left(r_{1}\right) E_{y}^{*}\left(r_{2}\right) ; \| . \\
E_{x}^{*}\left(r_{1}\right) E_{y}\left(r_{2}\right) ; & E_{y}\left(r_{1}\right) E_{y}^{*}\left(r_{2}\right) .
\end{array}\right\| .
$$

Taking into account Eq. (16), we rewrite the operator (17) as follows:

$$
\left\{\Phi\left(r_{1}, r_{2}\right)\right\}=\left\|\begin{array}{cc}
E_{x}\left(r_{1}\right) E_{x}\left(r_{2}\right) ; & E_{x}\left(r_{1}\right) E_{y}\left(r_{2}\right) \exp \left(i \delta_{2}\left(r_{2}\right)\right) ; \| . \\
E_{y}\left(r_{1}\right) E_{x}\left(r_{2}\right) \exp \left(i \delta_{1}\left(r_{1}\right)\right) ; & E_{y}\left(r_{1}\right) E_{y}\left(r_{2}\right) .
\end{array}\right\| .
$$

Basing on Eq. (18), it is possible to show that the DMP (3) for the two given points of the image is determined by the relation

$$
V^{2}\left(r_{1}, r_{2}\right)=\frac{\left(E_{x}\left(r_{1}\right) E_{x}\left(r_{2}\right)-E_{y}\left(r_{1}\right) E_{y}\left(r_{2}\right)\right)^{2}+4 E_{x}\left(r_{1}\right) E_{x}\left(r_{2}\right) E_{y}\left(r_{1}\right) E_{y}\left(r_{2}\right) \exp \left(i\left(\delta_{2}\left(r_{2}\right)-\delta_{1}\left(r_{1}\right)\right)\right)}{\left(E_{x}^{2}\left(r_{1}\right)+E_{y}^{2}\left(r_{1}\right)\right)\left(E_{x}^{2}\left(r_{2}\right)+E_{y}^{2}\left(r_{2}\right)\right)} .
$$

\section{The technique of DMP and singular states measurement for polarization inhomogeneous BT object fields}

The optical scheme for measuring coordinate distributions of the parameters of BT object fields is presented in Fig. 3. Illumination was performed with a collimated beam of HeNe laser $(\lambda=0.6328 \mu \mathrm{m}, \mathrm{W}=5.0 \mathrm{~mW}$ and the diameter $10 \mathrm{~mm})$. The polarization was controlled by quarter-wave plates 3 and 5 , while polarizer 4 was used to form a laser beam with the polarization azimuths $0^{\circ} \leq \alpha_{0} \leq 180^{\circ}$ and the ellipticities $0^{\circ} \leq \beta_{0} \leq 90^{\circ}$. Polarization images of BT obtained by means of micro-objective 7 were projected onto the array of CCD-camera 9 (800x600 pixels), which provided measurements of structural elements of the BT for the lateral scales from 2 to $2000 \mu \mathrm{m}$. The BT images were analyzed by means of polarizer 8 .

The technique for determining coordinate distribution of the polarization singularities and the DMP of the BT image consisted in the following steps:

1. The coordinate distribution of the intensities of the BT laser image $I\left(r_{m, n}\right)$ was measured by means of the CCD camera 10, without the analyzer 8 .

2. Analyzer 8 was then inserted, whose transmission axis was sequentially oriented at the angles of $\Theta=0^{\circ}$ and $\Theta=90^{\circ}$, and the intensity values $I^{(0)}\left(r_{m, n}\right) ; I^{(90)}\left(r_{m, n}\right)$ were measured.

3. After changing orientation of the transmission axis of analyzer within the range of $\Theta=0^{\circ} \rightarrow 180^{\circ}$, the minimal and maximal levels of the image intensity $I_{\min }\left(r_{m, n}\right) ; I_{\max }\left(r_{m, n}\right)$ for every pixel $(m, n)$ and the corresponding rotation angles $\left.\Theta\left(r_{m, n}\right)\right|_{I\left(r_{m, n}\right) \equiv I_{\min }}$ of the analyzer's transmission axis were measured. 


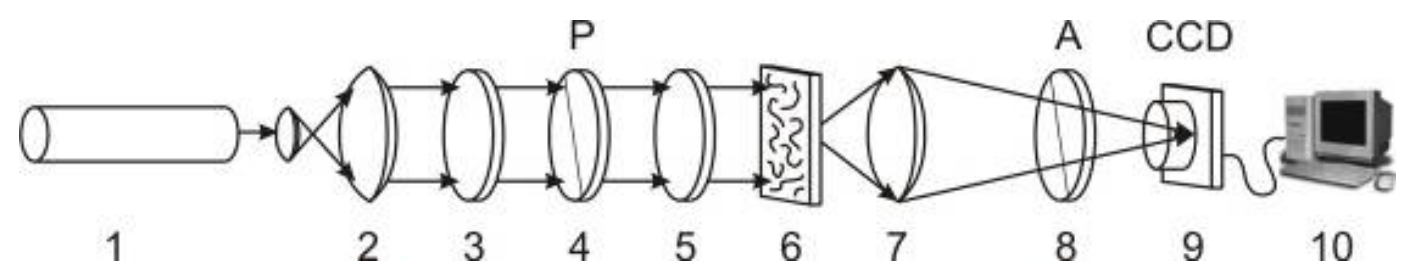

Fig. 3. Optical scheme for polarimetry of biological tissue histological samples. 1 - He-Ne laser; 2 - collimator; 3, 5 - quarter-wave plates; 4,8 - polarizers; 6 - object of investigation; 7 - microobjective; 9 - CCD camera; 10 - processor unit.

4. Coordinate distribution of the polarization states of light oscillations in the BT image was calculated according to the relations

$$
\alpha\left(r_{m, n}\right)=\left.\Theta\left(r_{m, n}\right)\right|_{I\left(r_{m, n}\right) \equiv I_{\min }}-\frac{\pi}{2}, \quad \beta\left(r_{m, n}\right)=\arctan \frac{I_{\min }\left(r_{m, n}\right)}{I_{\max }\left(r_{m, n}\right)}
$$

5. The local phase shifts $\delta_{1}\left(r_{1}\right)$ and $\delta_{2}\left(r_{2}\right)$ were calculated according with the relations

$$
\delta_{1}\left(r_{1}\right)=\arctan \left[\frac{\tan 2 \beta\left(r_{1}\right)}{\tan \alpha\left(r_{1}\right)}\right], \quad \delta_{2}\left(r_{2}\right)=\arctan \left[\frac{\tan 2 \beta\left(r_{2}\right)}{\tan \alpha\left(r_{2}\right)}\right] .
$$

6. The DMP of the coherent BT image was obtained by direct experimental measurements according to the relation

$$
\begin{aligned}
& \left|V^{2}\left(r_{n+k}, r_{n}\right)\right|=\frac{\left(\left(I^{(0)}\left(r_{n+k}\right) I^{(0)}\left(r_{n}\right)\right)^{\frac{1}{2}}-\left(I^{(90)}\left(r_{n+k}\right) I^{(90)}\left(r_{n}\right)\right)^{\frac{1}{2}}\right)^{2}}{I\left(r_{n+k}\right) I\left(r_{n}\right)}+ \\
& +\frac{4\left(I^{(0)}\left(r_{n+k}\right) I^{(90)}\left(r_{n+k}\right) I^{(0)}\left(r_{n}\right) I^{(90)}\left(r_{n}\right)\right)^{\frac{1}{2}} \cos \left(\delta_{n+k}\left(r_{n+k}\right)-\delta_{n}\left(r_{n}\right)\right)}{I\left(r_{n+k}\right) I\left(r_{n}\right)}
\end{aligned}
$$

Here $I^{(0)}\left(r_{i}\right)$ and $I^{(90)}\left(r_{i}\right)$ are the intensities of the BT image measured respectively for the orientations $\Theta=0^{\circ}$ and $\Theta=90^{\circ}$ of the transmission axis of the analyser.

7. The map of singly-degenerated (linearly polarized) singular points was obtained from the condition

$$
\beta\left(r_{m, n}\right)=0 .
$$

8. The map of doubly-degenerated (circularly polarized) singular points was obtained from the condition

$$
\beta\left(r_{m, n}\right)=\pi / 4
$$




\section{Objects of investigation and experimental results}

Initially, a histological section of myocardium tissue with the geometrical thickness of $100 \mu \mathrm{m}$ and a radial structure of anisotropic packing of myosin bundles was chosen for investigation (see Fig. 4). We chose this object in view of the fact that the opticalgeometric parameters of myocardium tissue are essentially close to the structure of virtual birefringent BT analyzed above (Fig. 1a). Thus, we would expect the most realistic conclusions based on the comparative analysis of topological structures of computermodelled and experimentally obtained polarization-inhomogeneous images.

The series in Fig. 5 presents the topological distributions of polarization singularities of S- and C-types obtained experimentally for the state of illumination $\left\{\alpha_{0}=0^{0} ; \beta_{0}=0^{0}\right\}$ and the fragment "P" of myocardium tissue image (see Fig. 4). Here the S-states are marked by black points, while the C-points with the right and left circulations respectively by red and blue points.

The obtained results reveal a presence of all types of the S-contours (Fig. 2) in the polarization image of myocardium tissue. Notice that Fig. 4 a presents the experimentally

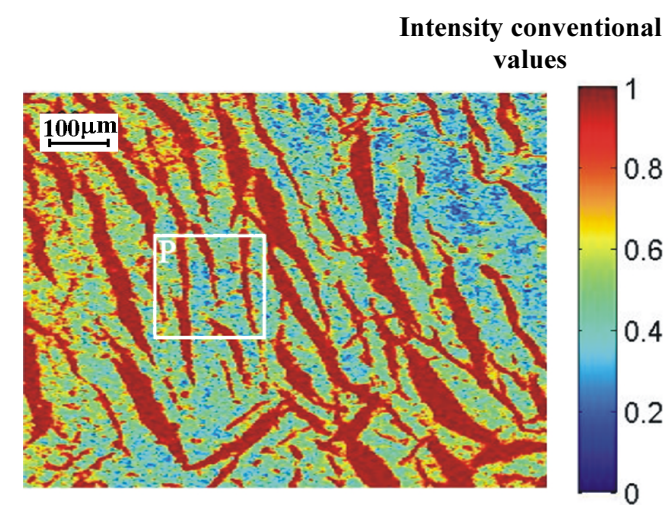

(a)

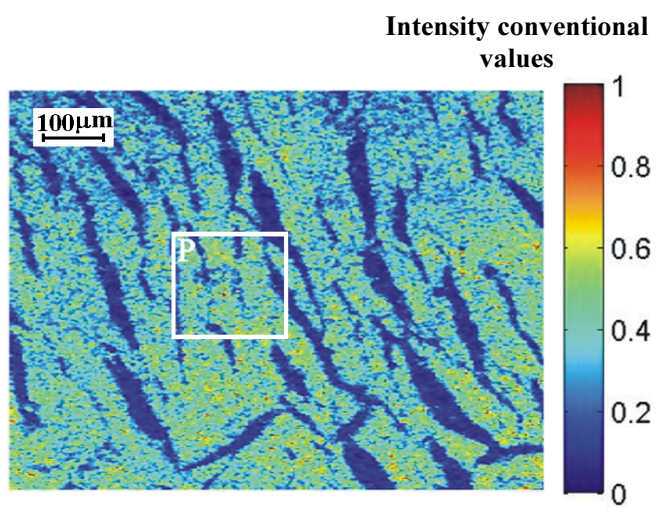

(b)

Fig. 4. Polarization images of myocardium tissue obtained in coaxial (a) and crossed (b) polarizer-analyzer. "P" depicts the area for topological polarization structure investigation.

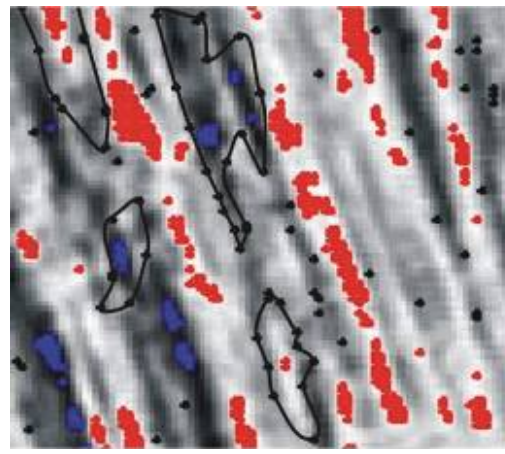

(a)

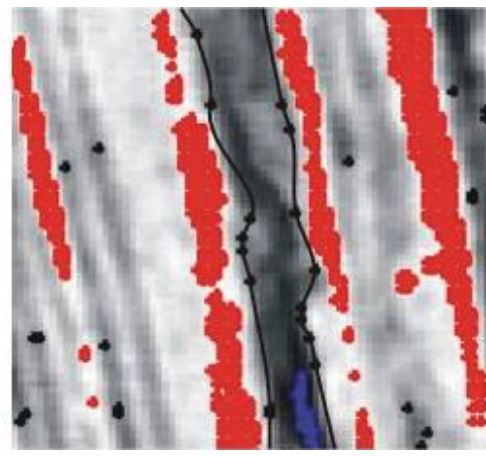

(b)

Fig. 5. Topological distributions of polarization singularities (S-contours) of myocardium tissue laser images. S-states are marked by black points; C-points with right circulation - red points; C-points with left circulation - blue points. 
revealed closed S-contours, which are analogous to the model structures presented in Fig. 2b. Fig. 4b shows topological structure of the unclosed S-contours, which are similar to the model structures presented in Fig. 2a. This can be related to the fact that optically anisotropic fibrils in the cross section of mono-polarized laser beam are differently oriented with respect to the polarization plane $\alpha_{0}$.

Similar results have been obtained for the other types of BT with birefringent islandlike architectonics, e. g. for the skin derma tissue (Fig. 6). The obtained data testify that the closed S-contours are the most typical for this type of objects. Therefore, one can state that the process of formation of the topological polarization structures of BT object fields, being a result of interrelation between the laser radiation and the birefringent crystallite structures, is identical to the process of formation of S-contours in the scattered radiation field due to statistical interference of differently polarized wave fronts [9-16].

On the other hand, polarization structure of the boundary field of BT highlights coordinate distribution of the properties of its phase-shift architectonics. It is known [1721] that orientation and phase structure of architectonic nets depend on physiological state of the BT. Thus, the interrelation between the topological singular structure of the boundary field of physiologically normal and pathologically changed skin derma (SD) should be expected.

In order to find this interrelation, we have studied histological sections of normal and oncologically changed SD samples. Polarizationally visualized architectonics of the SD is illustrated by microscopic images obtained for the case of crossed-polarizer geometry (see Fig. 7). Birefringent architectonics of healthy SD (Fig. 7a) is formed by statistically oriented bundles of collagen fibrils, the birefringence being $\Delta n \approx 10^{-3}$ [20]. The diameters of the fibrils are in the range $0.5-2 \mu \mathrm{m}$. The parallel fibril bundles form the fibres with the diameters ranging between 5 and $30 \mu \mathrm{m}$. Pathological changes of the SD are accompanied by increasing geometric sizes, both transverse and longitudinal, of the collagen fibres (see Fig. 7b). Fig. 8 shows coordinate distributions of the ellipticities $\beta(r)$ for the images of physiologically normal (Fig. 8a) and pathologically changed

(Fig. 8b) SD. The local values of the ellipticity $\beta(r)$ are obtained for the image sampling interval $\Delta r_{i}=1$ CCD-pixel and are given in the colour RGB scale.

The results show that the boundary field of the SD samples of both types is inhomogeneous with respect to polarization. The range of $\beta(r)$ changes is wide enough

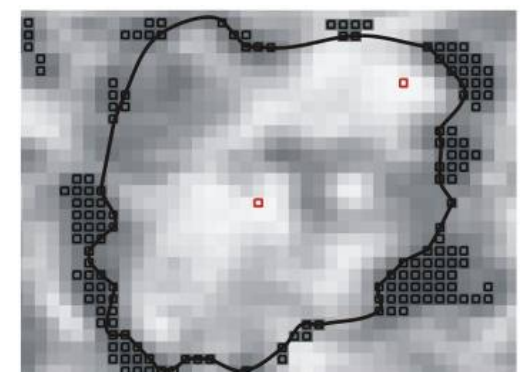

116
Fig. 6. Topological distributions of polarization singularities (S-contours) of skin derma tissue laser images. S-states are marked by black points; C-points with riaht circulation - red points. 

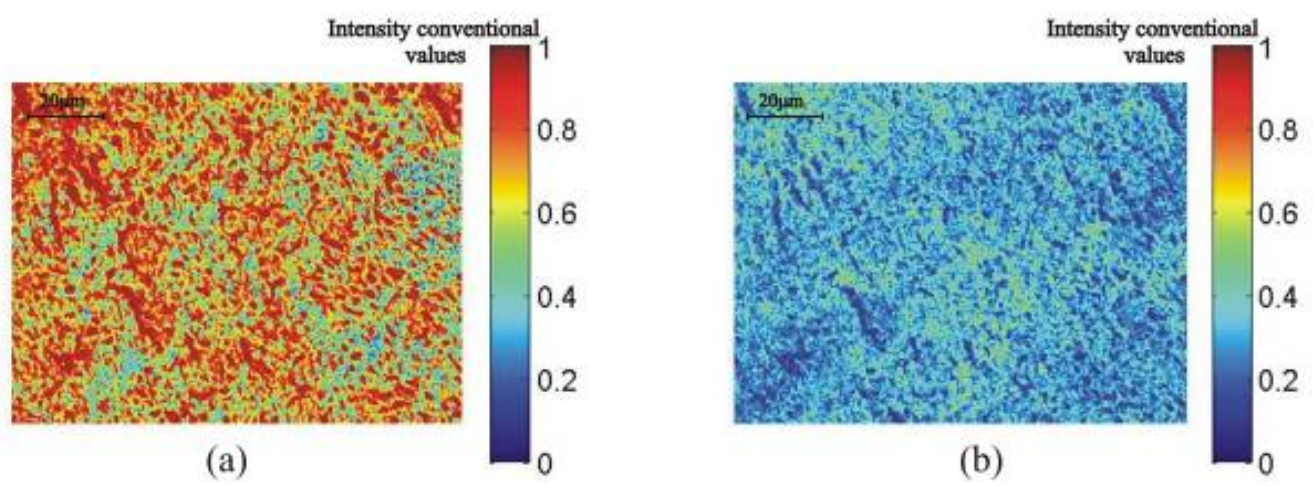

Fig. 7. Polarization images of skin derma tissue obtained in crossed-polarizer analyzer for physiologically normal condition (a) and oncologically changed condition (b).

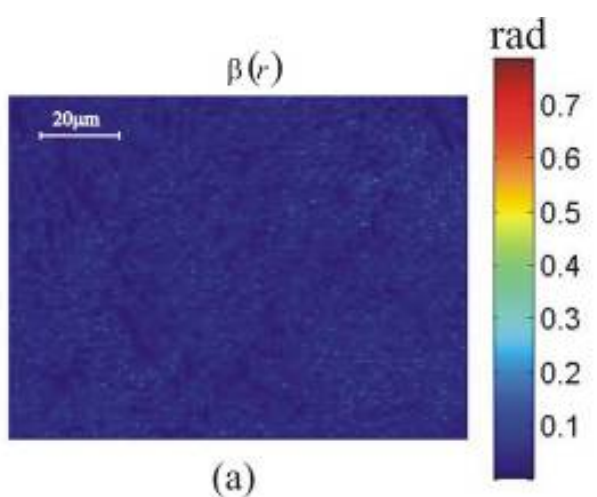

(a)

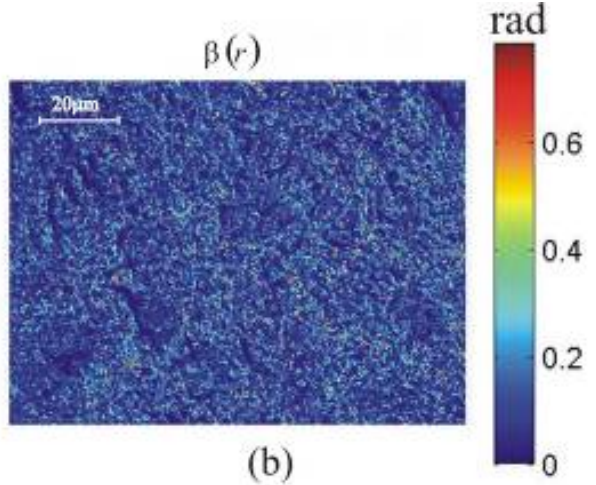

(b)

Fig. 8. Coordinate distributions of polarization ellipticities $\beta(x, y)$ of physiologically normal (a) and oncologically changed (b) skin derma images.

and spans the interval $-\pi / 4 \rightarrow \pi / 4$, including the polarization singularities that happen when $\beta(r)=0$ and $\beta(r)= \pm \pi / 4$. The main peculiarity of the polarization structures of the images is an increased geometric scale of $\beta(r)$ in the plane of pathologically changed SD image (see Fig. 8b). It could be associated with increasing the geometric size of the birefringent collagen structures.

In Fig. 9a, b we illustrate coordinate distribution of the polarization singularities of the SD images by the ensemble of green (linear states) and red (right and left circular states) points superimposed on the corresponding skin images. Besides of simply characterizing a presence of polarization inhomogeneities in the boundary field of birefringent SD samples, the obtained results can also serve as a basis for finding scenarios of forming such the inhomogeneity.

The detailed analysis of the coordinate distribution $\beta(r)=0$ and $\beta(r)= \pm \pi / 4$, obtained for the micro-objective magnification 7 up to $40 x$ (see Fig. 3) reveals mainly a closed "island" nature of S-contours in the boundary field (Fig. 10a, b). It shows also that the size of the S-contours in the healthy skin images lies in the range of $20-35 \mu \mathrm{m}$, while 
the cross size of the S-contours for the SD with oncological symptoms lies in the range $7-15 \mu \mathrm{m}$.

From the viewpoint of physics, formation of the S-contours and the circular states of light polarization is adequately described using ideas of the suggested model for the optical properties of birefringent collagen fibrils available in the SD architectonics. First, one can always find the fibres that have the orientation $(\rho)$ and the phase ( $\delta=2 \pi d \Delta n / \lambda$ ) parameters corresponding to the conditions which form the polarization singularities (cf. Eqs. (8)-(11)). Second, topological character of the S-contours and their geometric sizes are determined by the coordinate phase modulation $\delta(r)$ of a coherent beam by the birefringent $(\Delta n)$ fibre with the geometric size $d$. The calculations prove that the diameter of the S-contour for the collagen fibres with geometric cross-section $d=20 \mu \mathrm{m}$ is equal to $14 \mu \mathrm{m}$. The latter value correlates satisfactorily with the sizes of the S-contours determined experimentally for the healthy skin.

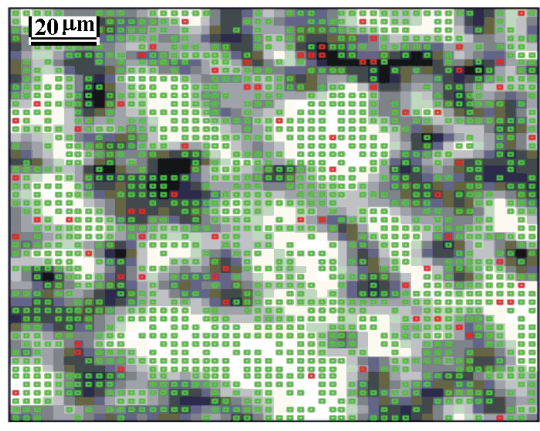

(a)

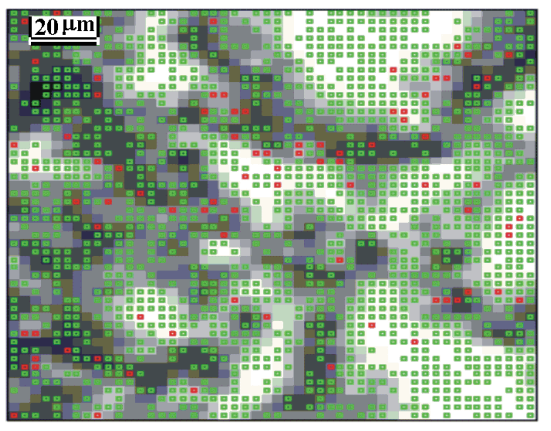

(b)

Fig. 9. Coordinate distributions of singly (green pixels) and doubly (red pixels) degenerated polarization singularities of physiologically normal (a) and oncologically changed (b) skin derma images. Such distribution is combined with skin derma image.

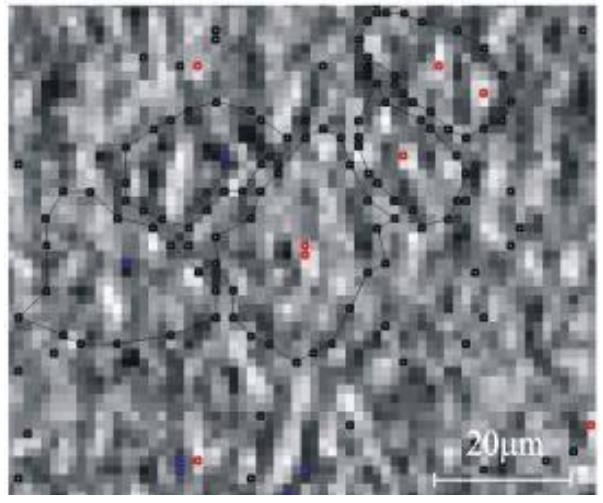

(a)

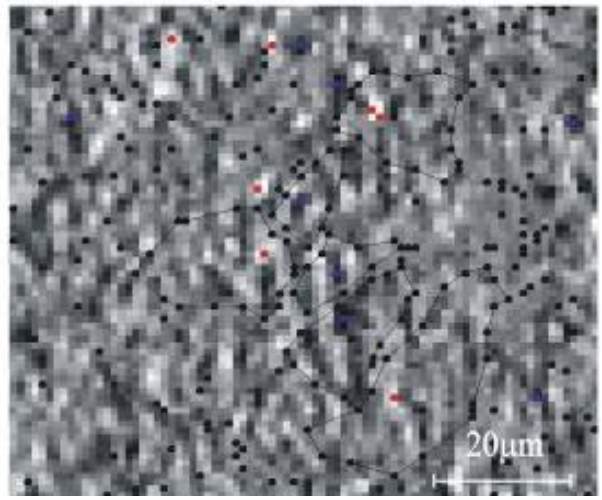

(b)

Fig. 10. Coordinate distributions of singly (green pixels $(\beta(r)=0)$, linear states) and doubly (red pixels, $\beta(r)=\pi / 4$, right circulation and blue pixels, $\beta(r)=-\pi / 4$, left circulation) degenerated polarization singularities of physiologically normal (a) and oncologically changed (b) skin derma images. 
Oncological changes are accompanied by an increase of geometrical size of the newly formed collagen fibres. We have calculated that the S-contour diameter is $9 \mu \mathrm{m}$ for the case of $d=35 \mu \mathrm{m}$. Taking this into account, we can relate a decrease in the S-contours size observed in the image of SD in pre-cancer state to increasing phase modulation depth of pathologically changed collagen fibres.

If the analysis of coordinate distribution of polarization singularities is regarded from a statistical point of view, it is necessary to consider sequentially the correlation interconnections between all the polarization states within the BT sample images. Such information is included in the DMP images of the investigated biological objects. According to Table 1, the DMP value $\left|V\left(\Delta r_{S}\right)^{2}\right|=0.5$ corresponds to the cross size of Scontour (the point of circular polarization surrounded by linear polarization points) [5]. That is why the parameter $\Delta r_{S}$ may be considered as a statistical lateral geometric size of the S-contours ensemble.

Thus, having determined coordinate distribution of the DMP for the BT image and calculated its autocorrelation function $G\left(\left|V^{2}\right|\right)$, one can derive the average lateral size $\Delta r_{S}$ of the S-contour, which corresponds to its half-width determined for the level $G\left(\left|V^{2}\right|\right)=0.5$. Coordinate distributions $\left|V^{2}(r)\right|$ calculated for the images of physiologically normal and pathologically changed SDs are presented in Fig. 11a and Fig. $11 \mathrm{~b}$, respectively. The distributions $\left|V^{2}(r)\right|$ for the images of SD samples of both types are coordinate-inhomogeneous. They are formed by the areas with both maximally similar (correlated) polarization states $\left(\left|V^{2}(r)\right| \rightarrow 1.0\right)$ and intermediate DMP values $\left(0.0 \leq\left|V^{2}(r)\right| \leq 1.0\right)$.

The investigated DMP structure for the SD images can be linked with different geometric structures of the collagen fibrils and their architectonics. An ordered nature of

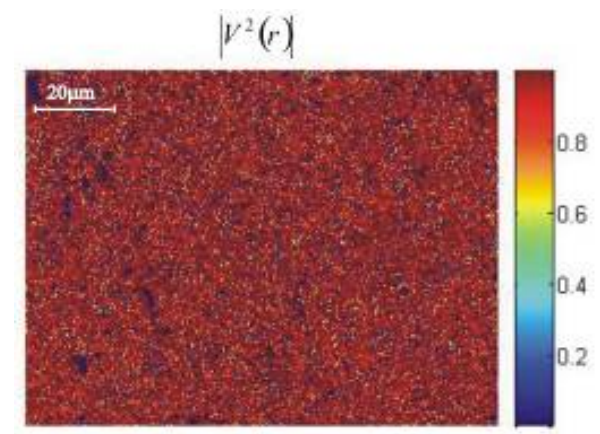

(a)

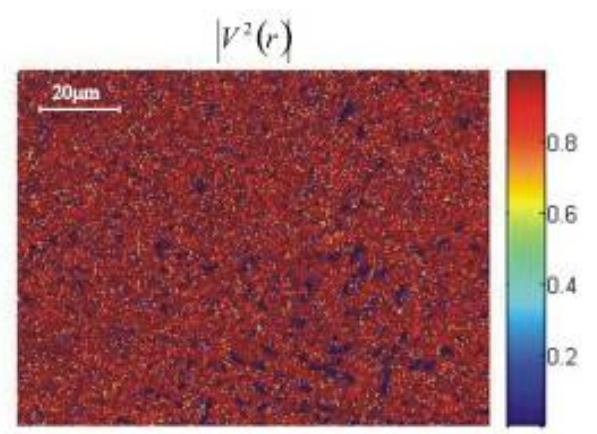

(b)

Fig. 11. Coordinate distributions of DMP values of physiologically normal (a) and oncologically changed (b) skin derma images. 
packing of the collagen fibrils within rather large geometric domains of their architectonic nets causes a high degree of correlation similarity $\left(V^{2}\left(\Delta r_{i}\right) \rightarrow 1\right)$ of polarization states at the corresponding points of their laser images. Passing into the other domains (i.e. the areas with different orientation structures of architectonics) is accompanied by changing polarization state and, correspondingly, decreasing DMP module at the points in a given part of the image. However, the DMP for the coherent BT image points becomes again extreme within the domain itself.

Pathological changes of the SD are morphologically entailed by an increase of geometric size of the fibrils in the collagen fibres and by formation of new directions of growth of the fibrils. From the standpoint of optics, this process is analogous to an increase in the angular interval between the polarization azimuth $\alpha_{0}$ of the laser beam illuminating SD and the fast axis direction $\rho\left(r_{m, n}\right)$ for optically anisotropic structures. At the corresponding points of the coherent SD image, different polarization states $\alpha\left(r_{m, n}\right), \quad \beta\left(r_{m, n}\right)$ of light oscillations are formed. Thus, the fluctuations of the DMP module increase. Such a coordinate modulation of $\left|V^{2}(r)\right|$ in the images of pathologically

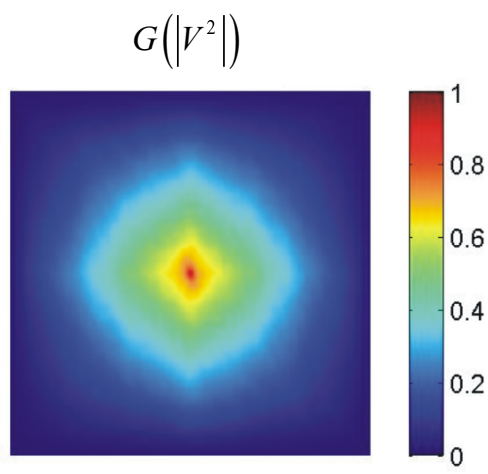

(a)

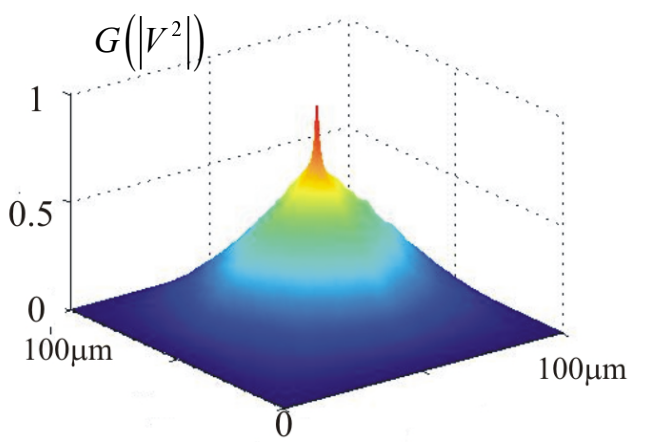

(c)

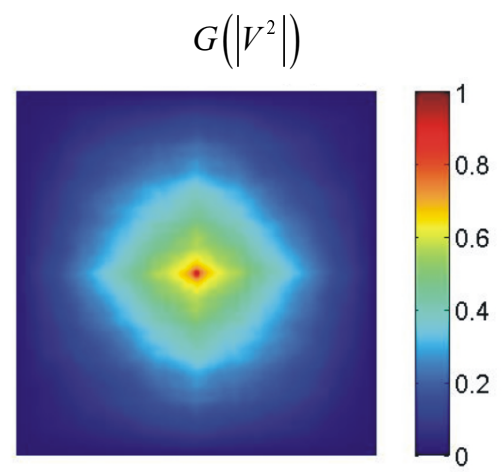

(b)

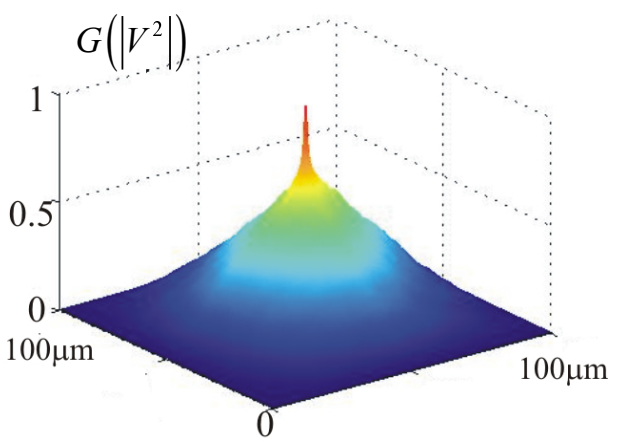

(d)

Fig. 12. Two-dimensional autocorrelation functions $(a, b)$ of DMP coordinate distributions. Fragments (c, d) present three-dimensional representation of corresponding autocorrelation functions. These results correspond to physiologically normal $(a, c)$ and pathologically changed (b, d) skin derma samples. 
changed SD must reveal itself in decreasing correlation radius or half-width $\Delta r_{S}$ of the corresponding autocorrelation function $G\left(\left|V^{2}\right|\right)$. Fig. 12 depicts the autocorrelation functions $G\left(\left|V^{2}\right|\right)$ derived from the coordinate distributions of DMP images for the cases of both healthy (Fig. 12a) and oncologically changed (Fig. 12b) SD. The data show clearly that a relationship between the half-width of the autocorrelation function and the S-contour sizes is close enough for the SD images of both types.

Thus, the main conclusion of this work is a statement about interconnection between the topological and statistic (correlation) approaches adopted for the description of mechanisms that form polarization inhomogeneity of the object fields. Namely, the statistical average size of an S-contour for such the fields should coincide with the halfwidth of autocorrelation function of the DMP.

\section{Conclusions}

1. The interconnection between the correlation structure and the topology of singular polarization states has been found for different points of polarization-inhomogeneous object field of BT:

- the boundary field formed by interaction of laser radiation with birefringent structures of BT is polarization-inhomogeneous and reveals singular states, whose topological structure corresponds to that of the S-contours resulting from statistical interference of partial wave fronts of the object field in different diffraction zones;

- the half-width of the autocorrelation function of DMP corresponds to the average statistical size of the S-contours.

2. The results obtained for the correlation of local polarization states in the laser images of BT may be used when diagnosing changes in the optic axis orientations and the phase shifts of birefringent biological crystals available in human tissue architectonics. This information may become basic for early pre-clinical diagnostics of pathological changes in the BT.

\section{References}

1. Stokes GG, 1852. On the composition and resolution of streams of polarized light from different sources. Trans. Cambridge Phil. Soc. 9: 399.

2. Born M. and Wolf E. Principles of optics. New York: Cambridge University Press (1999).

3. Ushenko A.G. and Pishak V.P., Laser Polarimetry of Biological Tissue. Principles and Applications. In Coherent-Domain Optical Methods. Biomedical Diagnostics, Environmental and Material Science (V. Tuchin, ed.). Kluwer Academic Publishers. 93-136 (2004).

4. Gori F, 1998. Matrix treatment for partially polarized, partially coherent beams. Opt. Lett. 23: 241-243.

Ukr. J. Phys. Opt. 2007, V8, №2 
5. Ellis J and Dogariu A, 2004. Complex degree of mutual polarization. Opt. Lett. 29: 536-538.

6. Benoit B. Mandelbrot. The Fractal Geometry of Nature. San Francisko: Freeman (1982).

7. Angelsky OV, Tomka YuYa, Ushenko AG, Ushenko YeG and Ushenko YuA, 2005. Investigation of 2D Mueller matrix structure of biological tissues for pre-clinical diagnostics of their pathological states. J. Phys. D Appl. Phys. 38: 4227-4235.

8. Mokhun I.I. Introduction to linear singular optics. In Correlation Optics: Application and Techniques (Ed. by O.V. Angelsky). SPIE Press, will be published.

9. Berry MV and Dennis MR, 2001. Polarization singularities in isotropic random vector waves. Proc. Roy. Soc. A457: 141-155.

10. Dolgov AD, Doroshkevich AG, Novikov DI and Novikov ID, 1999. Classification of singular points in the polarization of the cosmic microwave background and eigenvectors of the Stokes matrix. JETP Lett. 69: 427-433.

11. Konukhov AI and Melnikov LA, 2001. Optical vortices in a vector fields: the general definition based on the analogy with topological solitons in a 2D ferromagnet, and examples from the polarization transverse patterns in a laser. J. Opt. B: Quantum Semiclass. Opt. 3: S139-S144.

12. Freund I, 2001. Poincare vortices. Opt. Lett. 26: 1996-1998.

13. Nye JF, 1983. Lines of circular polarization in electromagnetic wave fields. Proc. Roy. Soc. A389: 279-290.

14. Freund I, 2004. Coherency matrix description of optical polarization singularities. J. Opt. A.: Pure and Appl. Opt. 6: S229-S234.

15. Angelsky OV, Mokhun II, Mokhun AI and Soskin MS, 2002. Interferometric methods in diagnostics of polarization singularities. Phys. Rev. E. 65: 036602.

16. Angelsky OV, Mokhun A, Mokhun I and Soskin M, 2002. The relationship between topological characteristics of component vortices and polarization singularities. Opt. Com. 207: 57-65.

17. Angelsky OV, Ushenko AG, Ermolenko SB, Burcovets DN, Pishak VP and Ushenko YuA, 2000. Polarization-Based Visualization of Multifractal Structures for the Diagnostics of Pathological Changes in Biological Tissues. Opt. Spectr. 89: 799-804.

18. Angelsky OV, Demyanovsky GV, Ushenko AG, Burcovets DN and Ushenko YuA, 2004. Wavelet analysis of two-dimensional birefringence images of architectonics in biotissues for diagnosing pathological changes. J. Biomed. Opt. 9: 679-690.

19. Angelsky OV, Ushenko AG, Burcovets DN and Ushenko YuA, 2005. Polarization visualization and selection of biotissue image two-layer scattering medium. J.

Biomed. Opt. 10: 014010. 
20. Angelsky OV, Ushenko AG and Ushenko YuA, 2005. Polarization reconstruction of orientation structure of biological tissues birefringent architectonic nets by using their Mueller-matrix speckle-images. J. Holography Speckle 2: 72-79.

21. Angelsky OV, Ushenko AG, Ushenko YuA, Ushenko YeG, Tomka YuYa, and Pishak VP, 2005. Polarization-correlation mapping of biological tissue coherent images. J. Biomed. Opt. 10: 064025. 